\title{
The social network of PELP1 and its implications in breast and prostate cancers
}

\author{
Vijay K Gonugunta, Lu Miao, Gangadhara R Sareddy', Preethi Ravindranathan, \\ Ratna Vadlamudi' and Ganesh V Raj
}

Department of Urology, UT Southwestern Medical Center at Dallas, 5323 Harry Hines Boulevard J8130, Dallas, Texas 75390, USA

${ }^{1}$ Department of Obstetrics and Gynecology, UT Health Science Center, San Antonio, Texas, USA
Correspondence should be addressed to G V Raj Email ganesh.raj@ utsouthwestern.edu

\begin{abstract}
Proline, glutamic acid- and leucine-rich protein 1 (PELP1) is a multi-domain scaffold protein that serves as a platform for various protein-protein interactions between steroid receptors (SRs) and signaling factors and cell cycle, transcriptional, cytoskeletal, and epigenetic remodelers. PELP1 is known to be a coregulator of transcription and participates in the nuclear and extranuclear functions of SRs, ribosome biogenesis, and cell cycle progression. The expression and localization of PELP1 are dysregulated in hormonal cancers including breast and prostate cancers. This review focuses on the interactive functions and therapeutic and prognostic significance of PELP1 in breast and prostate cancers.
\end{abstract}

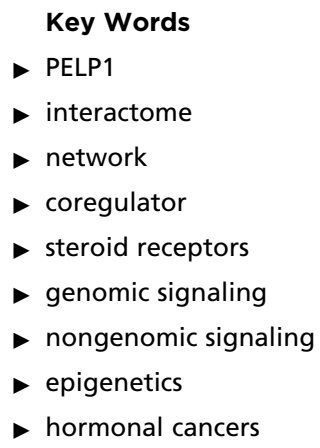

Endocrine-Related Cancer (2014) 21, T79-T86

\section{Introduction}

Proline-, glutamic acid- and leucine-rich protein 1 (PELP1), also referred to as modulator of nongenomic activity of estrogen receptor (MNAR) (Greger et al. 2006), is a promiscuous coregulator of steroid receptors (SRs) and exhibits corepressor or coactivator activity (Choi et al. 2004). PELP1 may be classified as a scaffolding protein (Vadlamudi \& Kumar 2007), and it serves as a 'mediator' for protein-protein interactions in different cellular processes such as cell cycle regulation, transcription, cytoskeletal and epigenetic modifications, and ribosome biogenesis (Choi et al. 2004, Nair et al. 2007, 2010a, Chakravarty et al. 2010a, 2011, Gonugunta et al. 2011, Mann et al. 2013). PELP1 is widely expressed in various tissues, with the highest levels being measured in the brains, testes, ovaries, and uteri of mice (Vadlamudi et al. 2001). Dysregulation of PELP1 expression has been observed in breast, ovarian, endometrial, brain, and prostate cancers (Dimple et al. 2008, Chakravarty et al. 2011, Cortez et al. 2012, Kefalopoulou et al. 2012, Wan \& Li 2012, Yang et al. 2012, Ravindranathan et al. 2013). PELP1 is involved in both genomic and extranuclear signaling pathways (Boonyaratanakornkit 2011, Girard et al. 2013, Renoir et al. 2013). The functions of PELP1 can be attributed to its multiple unique structural domains, which allow it to bind to different SRs to modulate their activity (Vadlamudi \& Kumar 2007, Girard et al. 2013). This review focuses on the structure, function, and genomic and extranuclear signaling of PELP1, associated signaling pathways, and its role as a target for therapeutic modulation in breast and prostate cancers. 


\section{PELP1 structural features}

The PELP1 gene is located on chromosome 17 on the short arm at region 13.2. The cDNA-translated protein comprises 1130 amino acids with a pI value of 4.3. However, due to the high content of proline and glutamic acid, the protein migrates as a $160 \mathrm{kDa}$ form on SDS-PAGE gels (predicted $120 \mathrm{kDa}$ ) (Vadlamudi \& Kumar 2007). Two alternatively spliced isoforms of PELP1 have been reported: a long $(3.9 \mathrm{~kb})$ form and a short $(3.5 \mathrm{~kb})$ form, both of which map to the same chromosomal region, 17p13.2, and encode the same protein. The longer isoform represents an immature transcript that contains an additional $435 \mathrm{bp}$ intronic region. Of the two, the short isoform is widely expressed in patients with hormonally driven cancers (Vadlamudi et al. 2001, Balasenthil \& Vadlamudi 2003).

PELP1 protein has multiple structural domains or discrete structural units made up of a complex of secondary structures such as $\alpha$-helices and $\beta$-sheets. These short peptide recognition domains may function in an independent context and enable the binding of PELP1 to different steroid hormone receptors and proteins. These domains may be found in multiple copies, but each domain may have its own unique interactome, depending on the structural context in which it is presented. Multiple domains may be involved in the binding between PELP1 and a specific protein partner: in these interactions, each PELP1 domain independently interacts with the target protein and cooperatively increases both its binding affinity to the target protein and the likelihood of PELP1-target protein complex formation. This built-in redundancy ensures interaction between PELP1 and critical protein cofactors. Furthermore, each PELP1 domain may modulate the activity and function of other domains on PELP1 and thus influence PELP1-protein interactions by modifying the secondary structure of the PELP1 domain. Proteins that bind to a PELP1 domain may in turn directly or indirectly interact with other proteins that bind to another domain on PELP1: thus PELP1 serves as a scaffolding protein enabling large-scale protein-protein interactions. The interactive domains on PELP1 thus promote cross talk between both proteins and signaling pathways. By virtue of its large interactome, PELP1 plays a critical role in cell signaling and cellular processes.

A domain scan using the online tool (http://scansite. mit.edu) has revealed several protein-interacting domains on PELP1 including multiple copies of the nuclear receptor (SR) box-interacting domains (LxxLL domains: $\mathrm{L}$ is leucine; $\mathrm{x}$ is any residue), $\operatorname{PxxP}(\mathrm{P}=$ proline) domains, SH2 domains, a long unusual stretch (70 amino acids) of glutamic acids flanked by two proline-rich regions as well as three nucleolar domains, and a single nuclear localization sequence (Fig. 1; Girard et al. 2013) Each of these regions is described in greater detail below.

\section{LxxLL domains}

The acidic LxxLL domain is conserved in more than 300 coregulators and is a known hot spot for SR interactions (Fuchs et al. 2013). Although the LxxLL domains have an $\alpha$-helical propensity, many factors, including the proper presentation of the LxxLL surface in the 3D structure, the availability of the domains for interaction, and the composition of the residues flanking the LxxLL core domain contribute to the determination of binding affinity and enable discrimination between binding partners. PELP1 has ten distinct LxxLL domains with unique flanking residues and structural presentation (Vadlamudi \& Kumar 2007). The interaction with SRs is typically enhanced by ligand binding, which induces conformational changes in the helix 12 (H12) region with the activation function 2 (AF-2) domain of the receptor facilitating the interaction with the short-helix LxxLL

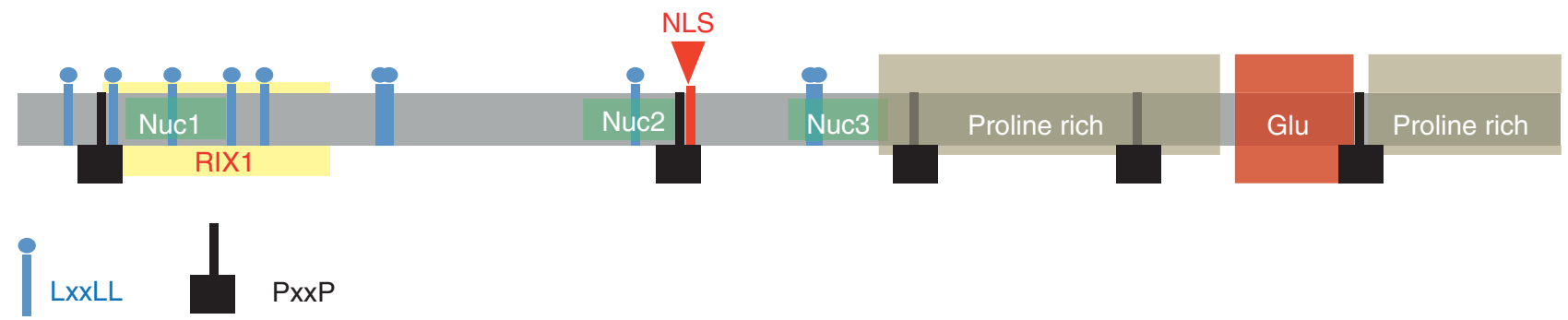

Figure 1

Schematic representation of the domains and sequences of PELP1 primary structure. PELP1 has ten LxxLL domains (blue lollipops), five PxxP domains (black hanging boxes), one nuclear localization sequence (NLS, red funnel), three nucleolar domains (Nuc1, Nuc2, and Nuc3, green boxes), and a glutamic-acid-rich region (Glu, dark red square) flanked by two proline-rich regions (ash-colored boxes) at its C-terminal end. http://erc.endocrinology-journals.org DOI: $10.1530 / E R C-13-0502$
(C) 2014 Society for Endocrinology Printed in Great Britain
Published by Bioscientifica Ltd 
domain (Folkertsma et al. 2007). The LxxLL domains of PELP1 have distinct binding specificities: for example, estrogen receptor-alpha $(\mathrm{ER} \alpha)$ preferentially interacts with the fourth and fifth LxxLL domains to mediate PELP1 signaling (Barletta et al. 2004). Although the specific LxxLL domains of PELP1 that interact with androgen receptor (AR), progesterone receptor (PR), glucocorticoid receptor (GR), or other SRs have not been characterized, they are distinct from the fourth LxxLL domain of PELP1 (Barletta et al. 2004, Yang et al. 2012, Ravindranathan et al. 2013).

\section{PxxP domains}

The PxxP domains were initially described as high-affinity binding sites for proteins with a SRC homology 3 (SH3) motif. The PxxP domains are often clustered in proline-rich stretches of proteins, and the bulky prolines are important for interaction with the SH3 motif, with its characteristic $\beta$-barrel fold of five to six $\beta$-strands being arranged in two tightly packed anti-parallel $\beta$-sheets (Vanhaesebroeck et al. 2001). Residues flanking the core PxxP domain may enhance the stability and binding specificity of proteins with SH3 domains, by forming additional contacts with the binding partner. Mechanistic studies have shown that PELP1 interacts with the SH3 motif of c-Src via its N-terminal PxxP domain and ER interacts with the SRC homology 2 (SH2) motif of Src at phosphotyrosine 537; the PELP1-ER interaction further stabilizes this trimeric complex, leading to the activation of the Src kinase, further activating the Ras/MAPK pathway (Barletta et al. 2004).

\section{SH2 domains}

Several SH2-binding domains within PELP1 may enable its interaction with kinases such as Abl and Src. The SH2 domains are complex structures with an anti-parallel $\beta$-sheet between flanking $\alpha$-helices, which results in a positively charged $\beta$-sheet pocket and enables highaffinity physical interactions with target proteins containing short peptides with phosphorylated tyrosines. Interestingly, the $\mathrm{N}$ - and $\mathrm{C}$-termini of the $\mathrm{SH} 2$ domains are very close to each other, enabling their introduction without disturbing the existing folding of the host protein. Tyrosine phosphorylation of PELP1 may recruit SH2 effectors: the phosphorylation of PELP1 at Tyr920 by Src kinase creates a binding site for the $\mathrm{SH} 2$ domain of p85 subunit of PI3K, leading to the activation of the PI3K pathway (Barletta et al. 2004, Dimple et al. 2008). Furthermore, the SH2 domains on PELP1 enable its interaction with STAT3 (Manavathi et al. 2005).

\section{Glutamic-acid-rich region}

PELP1 has an unusual C-terminal stretch of glutamic acids (amino acids 887-960), with 61/74 glutamic acid residues and $4 / 74$ aspartic acid residues. This sequence has a pI value of 2.18, making it an extremely acidic stretch. Unsurprisingly, this glutamic-acid-rich region is optimal for the interaction of PELP1 with basic-amino-acid-rich chromatin proteins such as histones $\mathrm{H} 1$ and $\mathrm{H} 3$ (Nair et al. 2004). Through the glutamic acid-rich region, PELP1 plays a role in chromatin remodeling by displacing histone $\mathrm{H} 1$ and by blocking hypoacetylated histones $\mathrm{H} 3$ and $\mathrm{H} 4$ from becoming the substrates of histone acetyltransferases (Choi et al. 2004). Furthermore, the glutamic-acid-rich region of PELP1 can function as a reader of histone dimethyl modification and facilitates the recruitment of histone demethylases such as lysine demethylase 1 (KDM1) to the DNA (Nair et al. 2010b). The glutamic-acid-rich region of PELP1 is further flanked on both sides by prolinerich domains, which may enable further recruitment and interaction with additional chromatin-modifying enzymes.

\section{Nucleolar and RIX1 domains}

PELP1 has three nucleolar domains (Nuc1: amino acids 79-160; Nuc2: amino acids 423-489; and Nuc 3: amino acids 569-642), which are important not only for the nuclear and nucleolar localization of PELP1, but also for rRNA processing and the activation of rDNA transcription and subsequent ribosome biogenesis (Gonugunta et al. 2011). PELP1 has a single RIX1 (rRNA processing/ribosome biogenesis) (amino acids 73-224) domain that enables complex formation with proteins IPI1 (TEX10), IPI3 (WDR18), and LASL1 and allows Rea1 AAA ATPase to associate with the $60 \mathrm{~S}$ ribosomal subunit, a critical step in rRNA processing and ribosome assembly. PELP1 is also a component of the SENP3 complex, a key complex in rRNA processing (Finkbeiner et al. 2011a,b).

\section{PELP1 signaling}

With respect to subcellular localization, PELP1 is expressed in both the nuclei and nucleoli of cells, but at lower amounts in the cytosol and plasma membrane (Vadlamudi et al. 2001, 2004, 2005, Dou et al. 2005, Gonugunta et al. 2011). PELP1 is involved in several critical cellular functions, including DNA and histone modification, ribosome synthesis, and genomic and nongenomic signaling. PELP1 is modified by several posttranslational mechanisms including phosphorylation

Published by Bioscientifica Ltd. 
(Vadlamudi \& Kumar 2007), glutamylation (Kashiwaya et al. 2010), and SUMOylation (Finkbeiner et al. 2011a). Several cytosolic and nuclear kinases such as EGFR, Src, PKA, ATM, Cdk2, and Cdk4 have been reported to phosphorylate PELP1 (Girard et al. 2013). The association of PELP1 with SRs in hormonal cancers has been explored extensively, and it forms the molecular basis of much of the initial interest in PELP1.

\section{Interaction with SRs}

The interactions of PELP1 with SRs such as ER $\alpha / \beta, A R, G R$, $\mathrm{PR}$, and vitamin D3 and mineralocorticoid (MR) receptors (Vadlamudi et al. 2001, Kayahara et al. 2008, Yang et al. 2012) involve the SR ligand-binding domain, and the binding is significantly enhanced by the addition of ligand. PELP1, by virtue of its function as a scaffolding protein, enables cross talk between different SRs (e.g., between AR and ER) and between SRs and a variety of cellular proteins involved in histone and DNA modification (e.g., HDAC2 and KDM1 (KDM1A), as shown for ER in breast cancer cells) (Nair et al. 2010b). PELP1 is a critical coregulator of the transcriptional activity of SRs.

\section{Role in genomic signaling}

PELP1 does not have a known DNA-binding domain and nor does it bind to DNA directly or function as a canonical transcriptional coactivator. Nonetheless, it plays a critical role in genomic regulation induced by SRs by virtue of its recruitment of coregulators. PELP1 enables cross talk between SRs including ER $\alpha / \beta, \mathrm{PR}$, and AR (Yang et al. 2012). Data from our laboratory demonstrated that PELP1 acts as a bridge between $\mathrm{ER} \alpha$ and $\mathrm{AR}$ to activate AR-responsive genes in the absence of androgen (Yang et al. 2012). Interestingly, PELP1 binds to AR and ER $\alpha$ through distinct LxxLL domains (Haas et al. 2005). In addition, recent data indicate that PELP1 may be involved in AR nuclear trafficking upon the activation of ligand (Ravindranathan et al. 2013).

Through distinct structural domains, PELP1 binds to critical cofactors involved in the modulation of chromatin structure and cooperatively influences gene expression (Choi et al. 2004, Gururaj et al. 2007, Nair et al. 2010b, Mann et al. 2013). For example, through its N-terminal LxxLL domain, PELP1 interacts with HDAC2 to repress chromatin decondensation (Choi et al. 2004). The C-terminal glutamic-acid-rich region enables the binding of PELP1 to hypoacetylated histones $\mathrm{H} 3$ and $\mathrm{H} 4$ to prevent further acetylation (Choi et al. 2004) and with
KDM1 to remove inhibitory histone methyl marks (Nair et al. 2010b). Furthermore, at the ER $\alpha$ target gene promoters, the association of PELP1 with CARM1 enhances its transactivation function by altering the histone $\mathrm{H} 3$ arginine methylation status (Mann et al. 2013). Together, these data clearly indicate that PELP1 has a role in the modulation of chromatin accessibility of critical target genes.

In addition, PELP1 interacts with several transcriptional activators including HDAC2, STAT3, SUMO2, MLL1/MLL, WDR5, SENP3, HCFC1/HCF1, RBBP5, ASH2L, and 5FMC complex to promote either optimal or aberrant conditions for the activity of SRs or transcription factors (Fanis et al. 2012).

Altogether, PELP1 is involved in SR-mediated genomic signaling both directly by interacting with SRs and their coregulators and indirectly by influencing the transcriptional milieu promoting the accessibility of SRs to the promoter.

\section{Role in nongenomic signaling}

PELP1 has no known enzymatic activity. However, PELP1 couples with SRs involved in efferent signaling pathways, through its ability to form complexes. PELP1 enables membrane-mediated signaling for membrane-bound ER $\alpha$ through the activation of cytosolic tyrosine kinase Src (c-Src) and PI3K in the cytosol via growth-factor-mediated membrane receptor signaling (Fig. 2; Barletta et al. 2004). The critical scaffolding function of PELP1 was confirmed in experiments using an estrogen dendrimer conjugate that uniquely activates ER extranuclear signaling and PELP1 knockdown, which blocks the activation of the ER-SrcPI3K-ILK1 pathway (Chakravarty et al. 2010b). PELP1mediated activation of cytosolic kinases in turn influences PELP1-influenced SR genomic function. For example, membrane-bound $\mathrm{ER} \alpha$ is able to activate c-Src by complexing with PELP1: c-Src subsequently phosphorylates and activates coactivator proteins in the cytoplasm, which then travel to the nucleus where they modulate PELP1-mediated $\mathrm{ER} \alpha$ transcriptional events (Barletta et al. 2004).

Similarly, PELP1 is critical for cytosolic ER-mediated nongenomic signaling (Fig. 2). Knockdown of PELP1 or mutation of its PxxP domain, critical for Src interaction, disrupts estradiol-induced activation of the MAP kinase pathway. PELP1 also interacts with STAT3 and enhances its activity in a Src-mitogen-activated protein kinasesensitive manner (Manavathi et al. 2005).

In addition, PELP1 interacts with several growth factor receptors such as EGFR and HER2 to mediate signaling cross talk with SRs (Vadlamudi et al. 2005). Collectively,

Published by Bioscientifica Ltd. 


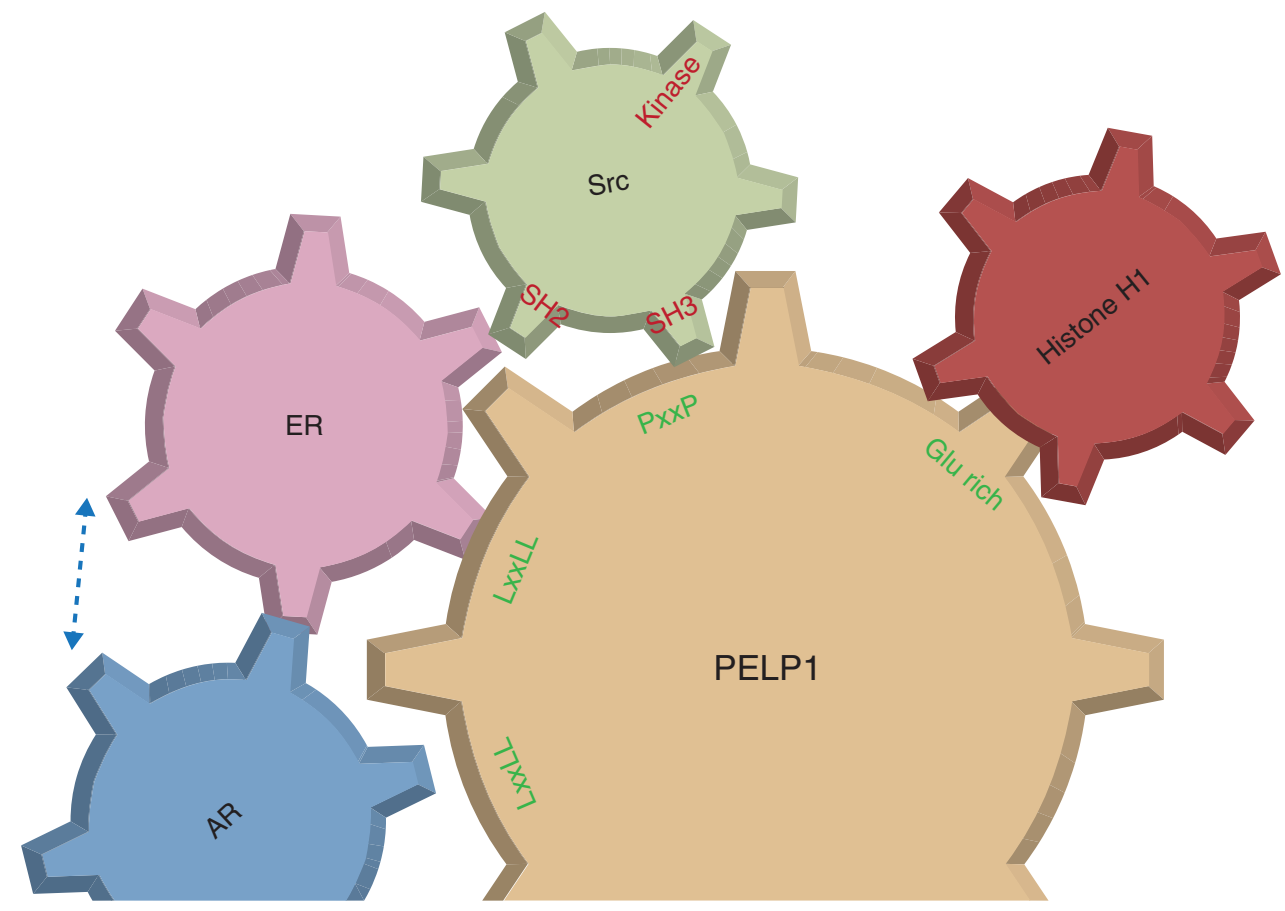

Figure 2

Schematic representation of the interactome of PELP1 through distinct domains: PELP1 functions as a scaffold enabling the interaction between various nuclear receptors and their cellular mediators. PELP1 interacts with SRs such as AR and ER through its LxxLL domains. PELP1 interacts with the SH3 motif on c-Src through its PxxP domain and through its SH2 domain:

these findings indicate that the primary role of PELP1 in nongenomic signaling is to enable the formation of multifunctional protein complexes that play critical roles in cell survival and migration and growth factor signaling cross talk with SRs.

\section{PELP1 in cancers}

The role of PELP1 as a key core coregulator of SR signaling has led to an examination of its role in hormonal cancers (Girard et al. 2013). The expression of PELP1 is dysregulated in a spectrum of hormonal cancers, including $60-80 \%$ of breast cancer cases, $60-70 \%$ of ovarian cancer cases, and $80-95 \%$ of prostate cancer cases, with higher expression profiles being correlated with adverse pathological features including more aggressive tumor types, higher tumor grades, and higher proliferative indices. The overexpression of PELP1 is correlated with adverse clinical outcomes such as nodal positivity, distant metastasis, and therapy resistance (Rajhans et al. 2007). In a large cohort of breast cancer specimens $(n=1162)$, the expression of PELP1 is an independent predictor of shorter breast-cancer-specific survival and shorter disease-free the involvement of two distinct domains of PELP1 is a built-in redundancy to ensure PELP1-c-Src interaction. Furthermore, c-Src and ER when docked on PELP1 may interact through the SH2 motif of c-Src. Finally, histones and histone-modifying enzymes interact with the Glu-rich motif on PELP1.

survival and its elevated expression is positively associated with poorer outcome (Habashy et al. 2010).

In aggressive, therapy-resistant breast and prostate cancers, PELP1 has been shown to play an important role in cell migration and metastasis via its ability to modulate miRNA, epithelial-to-mesenchymal transition (EMT), and mesenchymal-to-epithelial transition (MET) genes and mediate effective cross talk between SRs (Roy et al. 2013). The role of PELP1 in ribosome biogenesis also has implications in the progression of cancer by virtue of the importance of ribosomes in protein synthesis and cell growth and functioning. Defective signaling within these cellular processes may disrupt the physiological role of PELP1, contributing to oncogenic phenotypes, and these are discussed below.

\section{PELP1 in cell cycle progression}

Knockdown of PELP1 decreases the proliferation of both ER-positive and ER-negative breast cancer cells. Conversely, the overexpression of PELP1 is sufficient for cellular transformation and accelerated tumorigenesis. In Xenopus oocytes, PELP1 appears to mediate the inhibition of

Published by Bioscientifica Ltd. 
meiosis via $G \beta \gamma$ signaling (Haas et al. 2005). These data indicate that PELP1 functions as a proto-oncogene.

In breast cancer cells, PELP1 is important for estrogenmediated cell cycle progression to $S$ phase. The interaction of PELP1 with the cell-cycle-regulating protein retinoblastoma 1 (RB1) may be important for this cell cycle progression as PELP1 may sequester RB and relieve pRBmediated repression (Balasenthil \& Vadlamudi 2003). Furthermore, PELP1 is a cell-cycle-dependent kinase (CDK) substrate, is hyperphosphorylated during cell cycle progression, and recruited to $\mathrm{pRB} / \mathrm{E} 2 \mathrm{~F}$ target genes (Nair et al. 2010a).

\section{PELP1 in ribosome biogenesis}

The cross talk between ribosome biogenesis and cell division regulated by PELP1 has recently emerged as a key mechanism via its interaction with CDKs. CDK phosphorylation of PELP1 allows PELP1 to localize to the nucleolus, where it regulates rDNA transcription. The inhibition of CDKs dramatically reduces the localization of PELP1 to the nucleolus and has been shown to decrease rDNA promoter activity (Gonugunta et al. 2011). This indicates that PELP1 has an overarching role not only in SR-mediated gene regulation but also in cell division. As the nucleolus is seen as the hub of ribosomal DNA transcription and ribosomal subunit processing, increased expression of PELP1 in the nucleolus allows increased protein synthesis, cell survival, and tumorigenic phenotype levels (Finkbeiner et al. 2011a,b, Gonugunta et al. 2011, Castle et al. 2012).

\section{PELP1 and metastasis}

Enhanced expression of PELP1 has been correlated with increased motility and invasion of tumor cells (Chakravarty et al. 2010b, Roy et al. 2013). In contrast, knockdown of PELP1 results in decreased cell migration via downregulation of the ER $\alpha$-Src-PELP1-PI3K-ILK1 pathway, which disrupts cytoskeletal organization (Chakravarty et al. 2010b). The overexpression of PELP1 enhances EMT by regulating the expression of genes involved in EMT including MMPs, SNAIL (SNAI1), TWIST (TWIST1), ZEB (ZEB1), MYC, and MTA1 as well as miR-200a and miR-141 in breast cancer patients (Chakravarty et al. 2010b, Roy et al. 2012, 2013, Wan \& Li 2012).

\section{PELP1 and therapy resistance}

Several studies have indicated that the deregulation of PELP1 contributes to therapy resistance and that the knockdown of PELP1 or blockage of PELP1-mediated extranuclear signaling sensitizes cells to therapy (Vadlamudi etal. 2005, Nagpal et al. 2008, Kumar et al. 2009, Nair et al. 2011, Vallabhaneni et al. 2011). Interestingly, the subcellular localization of PELP1 is dysregulated in tumors with a cytosolic predominance in a subset of endometrial tumors, which exhibit resistance to tamoxifen anti-hormonal therapy. Patients whose tumors have high levels of cytoplasmic PELP1 respond poorly to tamoxifen therapy compared with patients whose tumors have low levels of cytoplasmic PELP1 (Kumar et al. 2009). These observations are in agreement with results from an experiment in which tamoxifen-susceptible MCF-7 cells engineered to express PELP1 in cytosol (by modification the nuclear localization sequence) were found to exhibit resistance to tamoxifen (Vadlamudi et al. 2005, Kumar et al. 2009, Gonugunta VK, Sareddy GR, Krishnan SR, Cortez V, Roy SS, Tekmal RR \& Vadlamudi RK, 2014, unpublished observations). The subcellular localization of PELP1 could be used as a biomarker of hormone sensitivity or vulnerability.

\section{Targeting PELP1 in hormonal cancers}

The role of PELP1 in a number of cellular processes and signaling pathways via its various domains makes PELP1 both an attractive and a daunting target for therapeutic modulation. Genetic intervention studies have shown PELP1 knockout to be lethal during embryonic development, which indicates the importance of PELP1 in development (Vadlamudi, unpublished data). Initial attempts targeted blocking PELP1 downstream signaling pathways such as the PELP1-Src axis, PELP1-CDK2 axis, and PELP1-KDM1 axis. The Src inhibitor dasatinib has been found to exhibit therapeutic utility in blocking the PELP1 signaling axis (Vallabhaneni et al. 2011). The CDK inhibitor roscovitine has been found to be effective at reducing the oncogenic processes mediated by PELP1 (Nair et al. 2011). KDM1 and CARM1 inhibitors have also been found to substantially inhibit tumorigenic functions of PELP1 (Cortez et al. 2012, Mann et al. 2013). Even though these studies have established the therapeutic potential of the PELP1 axis in treating hormone-relatedcancer patients, these drugs are not specific to PELP1 and new drugs directly targeting PELP1 interactions with increased specificity are required. One such strategy involves targeting specific functions of PELP1 as a coregulator and scaffolding protein. Our recent study has demonstrated the feasibility of targeting the interface between PELP1 and AR interactions with small peptidomimetics that compete for AR binding to

Published by Bioscientifica Ltd. 
PELP1 and effectively disrupt AR-PELP1 interactions (Ravindranathan et al. 2013). Consequently, the peptidomimetics result in decreased AR uptake into the nucleus, effectively blocking ligand-driven AR activation, with reduced expression of canonical AR-regulated genes, decreased cancer cell proliferation, and inhibition of tumor growth in xenograft and ex vivo cultures of primary prostate tumor cells (Ravindranathan et al. 2013). The results of these studies indicate that specific targeting of PELP1 in tumors may serve as a viable therapeutic strategy.

\section{Conclusions and future directions}

PELP1 functions as a scaffolding protein and enables critical protein-protein interactions due to its various structural domains. Although bereft of known enzymatic activity or ability to bind to DNA, PELP1 is a critical cellular protein that influences cellular signaling cascades and the cellular transcriptional machinery. Knockdown of PELP1 is lethal during embryonic development, indicating its importance in cellular functions. The overexpression of PELP1 portends a poor prognosis for patients with hormone-related cancers. Novel approaches to selectively disrupt the interaction of PELP1 with specific protein partners or to selectively knockdown PELP1 in tumor cells may enable effective targeting of cancers.

\section{Declaration of interest}

The authors declare that there is no conflict of interest that could be perceived as prejudicing the impartiality of the review.

\section{Funding}

This work was funded by the Department of Defense, the Prostate Cancer Foundation, and the James Thompson Research Foundation.

\section{References}

Balasenthil S \& Vadlamudi RK 2003 Functional interactions between the estrogen receptor coactivator PELP1/MNAR and retinoblastoma protein. Journal of Biological Chemistry 278 22119-22127. (doi:10.1074/ jbc.M212822200)

Barletta F, Wong CW, McNally C, Komm BS, Katzenellenbogen B \& Cheskis BJ 2004 Characterization of the interactions of estrogen receptor and MNAR in the activation of cSrc. Molecular Endocrinology 18 1096-1108. (doi:10.1210/me.2003-0335)

Boonyaratanakornkit V 2011 Scaffolding proteins mediating membraneinitiated extra-nuclear actions of estrogen receptor. Steroids $\mathbf{7 6}$ 877-884. (doi:10.1016/j.steroids.2011.02.017)

Castle CD, Cassimere EK \& Denicourt C 2012 LAS1L interacts with the mammalian Rix1 complex to regulate ribosome biogenesis. Molecular Biology of the Cell 23 716-728. (doi:10.1091/mbc.E11-06-0530)

Chakravarty D, Tekmal RR \& Vadlamudi RK 2010a PELP1: a novel therapeutic target for hormonal cancers. IUBMB Life 62 162-169. (doi:10.1002/iub.287)
Chakravarty D, Nair SS, Santhamma B, Nair BC, Wang L, Bandyopadhyay A, Agyin JK, Brann D, Sun LZ, Yeh IT et al. 2010 $b$ Extranuclear functions of ER impact invasive migration and metastasis by breast cancer cells. Cancer Research 70 4092-4101. (doi:10.1158/0008-5472.CAN-09-3834)

Chakravarty D, Roy SS, Babu CR, Dandamudi R, Curiel TJ, Vivas-Mejia P, Lopez-Berestein G, Sood AK \& Vadlamudi RK 2011 Therapeutic targeting of PELP1 prevents ovarian cancer growth and metastasis. Clinical Cancer Research 17 2250-2259. (doi:10.1158/1078-0432. CCR-10-2718)

Choi YB, Ko JK \& Shin J 2004 The transcriptional corepressor, PELP1, recruits HDAC2 and masks histones using two separate domains. Journal of Biological Chemistry 279 50930-50941. (doi:10.1074/ jbc.M406831200)

Cortez V, Mann M, Tekmal S, Suzuki T, Miyata N, Rodriguez-Aguayo C, Lopez-Berestein G, Sood AK \& Vadlamudi RK 2012 Targeting the PELP1-KDM1 axis as a potential therapeutic strategy for breast cancer. Breast Cancer Research 14 R108. (doi:10.1186/bcr3229)

Dimple C, Nair SS, Rajhans R, Pitcheswara PR, Liu J, Balasenthil S, Le XF, Burow ME, Auersperg N, Tekmal RR et al. 2008 Role of PELP1/MNAR signaling in ovarian tumorigenesis. Cancer Research $684902-4909$. (doi:10.1158/0008-5472.CAN-07-5698)

Dou Y, Milne TA, Tackett AJ, Smith ER, Fukuda A, Wysocka J, Allis CD, Chait BT, Hess JL \& Roeder RG 2005 Physical association and coordinate function of the H3 K4 methyltransferase MLL1 and the H4 K16 acetyltransferase MOF. Cell 121 873-885. (doi:10.1016/j.cell. 2005.04.031)

Fanis P, Gillemans N, Aghajanirefah A, Pourfarzad F, Demmers J, Esteghamat F, Vadlamudi RK, Grosveld F, Philipsen S \& van Dijk TB 2012 Five friends of methylated chromatin target of protein-argininemethyltransferase[prmt]-1 (chtop), a complex linking arginine methylation to desumoylation. Molecular and Cellular Proteomics 11 1263-1273. (doi:10.1074/mcp.M112.017194)

Finkbeiner E, Haindl M \& Muller S 2011 $a$ The SUMO system controls nucleolar partitioning of a novel mammalian ribosome biogenesis complex. EMBO Journal 30 1067-1078. (doi:10.1038/emboj.2011.33)

Finkbeiner E, Haindl M, Raman N \& Muller S 2011 $b$ SUMO routes ribosome maturation. Nucleus 2 527-532. (doi:10.4161/nucl.2.6.17604)

Folkertsma S, van Noort PI, de Heer A, Carati P, Brandt R, Visser A, Vriend G $\&$ de Vlieg J 2007 The use of in vitro peptide binding profiles and in silico ligand-receptor interaction profiles to describe ligand-induced conformations of the retinoid $\mathrm{X}$ receptor $\alpha$ ligand-binding domain. Molecular Endocrinology 21 30-48. (doi:10.1210/me.2006-0072)

Fuchs S, Nguyen HD, Phan TT, Burton MF, Nieto L, de Vries-van Leeuwen IJ, Schmidt A, Goodarzifard M, Agten SM, Rose R et al. 2013 Proline primed helix length as a modulator of the nuclear receptor-coactivator interaction. Journal of the American Chemical Society 135 4364-4371. (doi:10.1021/ja311748r)

Girard BJ, Daniel AR, Lange CA \& Ostrander JH 2013 PELP1: a review of PELP1 interactions, signaling, and biology. Molecular and Cellular Endocrinology 382 642-651. (doi:10.1016/j.mce.2013.07.031)

Gonugunta VK, Nair BC, Rajhans R, Sareddy GR, Nair SS \& Vadlamudi RK 2011 Regulation of rDNA transcription by proto-oncogene PELP1. PLoS ONE 6 e21095. (doi:10.1371/journal.pone.0021095)

Gonugunta VK, Sareddy GR, Krishnan SR, Cortez V, Roy SS, Tekmal RR \& Vadlamudi RK 2014 Inhibition of mTOR signaling reduces PELP1mediated tumor growth and therapy resistance. Molecular Cancer Therapeutics 13 1578-1588. (doi:10.1158/1535-7163.MCT-13-0877)

Greger JG, Guo Y, Henderson R, Ross JF \& Cheskis BJ 2006 Characterization of MNAR expression. Steroids 71 317-322. (doi:10.1016/j.steroids.2005. 09.016)

Gururaj AE, Peng S, Vadlamudi RK \& Kumar R 2007 Estrogen induces expression of BCAS3, a novel estrogen receptor- $\alpha$ coactivator, through proline-, glutamic acid-, and leucine-rich protein-1 (PELP1). Molecular Endocrinology 21 1847-1860. (doi:10.1210/me.2006-0514)

Haas D, White SN, Lutz LB, Rasar M \& Hammes SR 2005 The modulator of nongenomic actions of the estrogen receptor (MNAR) regulates 
transcription-independent androgen receptor-mediated signaling: evidence that MNAR participates in G protein-regulated meiosis in Xenopus laevis oocytes. Molecular Endocrinology 19 2035-2046. (doi:10.1210/me.2004-0531)

Habashy HO, Powe DG, Rakha EA, Ball G, Macmillan RD, Green AR \& Ellis IO 2010 The prognostic significance of PELP1 expression in invasive breast cancer with emphasis on the ER-positive luminal-like subtype. Breast Cancer Research and Treatment 120 603-612. (doi:10.1007/ s10549-009-0419-9)

Kashiwaya K, Nakagawa H, Hosokawa M, Mochizuki Y, Ueda K, Piao L, Chung S, Hamamoto R, Eguchi H, Ohigashi H et al. 2010 Involvement of the tubulin tyrosine ligase-like family member 4 polyglutamylase in PELP1 polyglutamylation and chromatin remodeling in pancreatic cancer cells. Cancer Research 70 4024-4033. (doi:10.1158/0008-5472. CAN-09-4444)

Kayahara M, Ohanian J, Ohanian V, Berry A, Vadlamudi R \& Ray DW 2008 MNAR functionally interacts with both NH2- and $\mathrm{COOH}$-terminal GR domains to modulate transactivation. American Journal of Physiology. Endocrinology and Metabolism 295 E1047-E1055. (doi:10.1152/ajpendo. 90429.2008)

Kefalopoulou Z, Tzelepi V, Zolota V, Grivas PD, Christopoulos C, Kalofonos H, Maraziotis T \& Sotiropoulou-Bonikou G 2012 Prognostic value of novel biomarkers in astrocytic brain tumors: nuclear receptor co-regulators AIB1, TIF2, and PELP1 are associated with high tumor grade and worse patient prognosis. Journal of Neuro-Oncology $\mathbf{1 0 6}$ 23-31. (doi:10.1007/s11060-011-0637-y)

Kumar R, Zhang H, Holm C, Vadlamudi RK, Landberg G \& Rayala SK 2009 Extranuclear coactivator signaling confers insensitivity to tamoxifen. Clinical Cancer Research 15 4123-4130. (doi:10.1158/1078-0432. CCR-08-2347)

Manavathi B, Nair SS, Wang RA, Kumar R \& Vadlamudi RK 2005 Proline-, glutamic acid-, and leucine-rich protein-1 is essential in growth factor regulation of signal transducers and activators of transcription 3 activation. Cancer Research 65 5571-5577. (doi:10.1158/0008-5472. CAN-04-4664)

Mann M, Cortez V \& Vadlamudi R 2013 PELP1 oncogenic functions involve CARM1 regulation. Carcinogenesis 34 1468-1475. (doi:10.1093/ carcin/bgt091)

Nagpal JK, Nair S, Chakravarty D, Rajhans R, Pothana S, Brann DW, Tekmal RR \& Vadlamudi RK 2008 Growth factor regulation of estrogen receptor coregulator PELP1 functions via protein kinase A pathway. Molecular Cancer Research 6 851-861. (doi:10.1158/1541-7786.MCR-07-2030)

Nair SS, Mishra SK, Yang Z, Balasenthil S, Kumar R \& Vadlamudi RK 2004 Potential role of a novel transcriptional coactivator PELP1 in histone H1 displacement in cancer cells. Cancer Research 64 6416-6423. (doi:10.1158/0008-5472.CAN-04-1786)

Nair SS, Guo Z, Mueller JM, Koochekpour S, Qiu Y, Tekmal RR, Schule R, Kung HJ, Kumar R \& Vadlamudi RK 2007 Proline-, glutamic acid-, and leucine-rich protein-1/modulator of nongenomic activity of estrogen receptor enhances androgen receptor functions through LIM-only coactivator, four-and-a-half LIM-only protein 2. Molecular Endocrinology 21 613-624. (doi:10.1210/me.2006-0269)

Nair BC, Nair SS, Chakravarty D, Challa R, Manavathi B, Yew PR, Kumar R, Tekmal RR \& Vadlamudi RK 2010a Cyclin-dependent kinase-mediated phosphorylation plays a critical role in the oncogenic functions of PELP1. Cancer Research 70 7166-7175. (doi:10.1158/0008-5472. CAN-10-0628)

Nair SS, Nair BC, Cortez V, Chakravarty D, Metzger E, Schule R, Brann DW, Tekmal RR \& Vadlamudi RK $2010 b$ PELP1 is a reader of histone H3 methylation that facilitates oestrogen receptor- $\alpha$ target gene activation by regulating lysine demethylase 1 specificity. EMBO Reports $\mathbf{1 1}$ 438-444. (doi:10.1038/embor.2010.62)

Nair BC, Vallabhaneni S, Tekmal RR \& Vadlamudi RK 2011 Roscovitine confers tumor suppressive effect on therapy-resistant breast tumor cells. Breast Cancer Research 13 R80. (doi:10.1186/bcr2929)

Rajhans R, Nair S, Holden AH, Kumar R, Tekmal RR \& Vadlamudi RK 2007 Oncogenic potential of the nuclear receptor coregulator proline-, glutamic acid-, leucine-rich protein 1/modulator of the nongenomic actions of the estrogen receptor. Cancer Research 67 5505-5512. (doi:10.1158/0008-5472.CAN-06-3647)

Ravindranathan P, Lee TK, Yang L, Centenera MM, Butler L, Tilley WD, Hsieh JT, Ahn JM \& Raj GV 2013 Peptidomimetic targeting of critical androgen receptor-coregulator interactions in prostate cancer. Nature Communications 4 1923. (doi:10.1038/ncomms2912)

Renoir JM, Marsaud V \& Lazennec G 2013 Estrogen receptor signaling as a target for novel breast cancer therapeutics. Biochemical Pharmacology 85 449-465. (doi:10.1016/j.bcp.2012.10.018)

Roy S, Chakravarty D, Cortez V, De Mukhopadhyay K, Bandyopadhyay A, Ahn JM, Raj GV, Tekmal RR, Sun L \& Vadlamudi RK 2012 Significance of PELP1 in ER-negative breast cancer metastasis. Molecular Cancer Research 10 25-33. (doi:10.1158/1541-7786.MCR-110456)

Roy SS, Gonugunta VK, Bandyopadhyay A, Rao MK, Goodall GJ, Sun LZ, Tekmal RR \& Vadlamudi RK 2013 Significance of PELP1/HDAC2/ miR-200 regulatory network in EMT and metastasis of breast cancer. Oncogene (In Press). (doi:10.1038/onc.2013.332)

Vadlamudi RK \& Kumar R 2007 Functional and biological properties of the nuclear receptor coregulator PELP1/MNAR. Nuclear Receptor Signaling 5 e004. (doi:10.1621/nrs.05004)

Vadlamudi RK, Wang RA, Mazumdar A, Kim Y, Shin J, Sahin A \& Kumar R 2001 Molecular cloning and characterization of PELP1, a novel human coregulator of estrogen receptor $\alpha$. Journal of Biological Chemistry $\mathbf{2 7 6}$ 38272-38279. (doi:10.1074/jbc.M103783200)

Vadlamudi RK, Balasenthil S, Broaddus RR, Gustafsson JA \& Kumar R 2004 Deregulation of estrogen receptor coactivator proline-, glutamic acid-, and leucine-rich protein-1/modulator of nongenomic activity of estrogen receptor in human endometrial tumors. Journal of Clinical Endocrinology and Metabolism 89 6130-6138. (doi:10.1210/ jc.2004-0909)

Vadlamudi RK, Manavathi B, Balasenthil S, Nair SS, Yang Z, Sahin AA \& Kumar R 2005 Functional implications of altered subcellular localization of PELP1 in breast cancer cells. Cancer Research $\mathbf{6 5}$ 7724-7732. (doi:10.1158/0008-5472.CAN-05-0614)

Vallabhaneni S, Nair BC, Cortez V, Challa R, Chakravarty D, Tekmal RR \& Vadlamudi RK 2011 Significance of ER-Src axis in hormonal therapy resistance. Breast Cancer Research and Treatment $130377-385$ (doi:10.1007/s10549-010-1312-2)

Vanhaesebroeck B, Leevers SJ, Ahmadi K, Timms J, Katso R, Driscoll PC, Woscholski R, Parker PJ \& Waterfield MD 2001 Synthesis and function of 3-phosphorylated inositol lipids. Annual Review of Biochemistry $\mathbf{7 0}$ 535-602. (doi:10.1146/annurev.biochem.70.1.535)

Wan J \& Li X 2012 PELP1/MNAR suppression inhibits proliferation and metastasis of endometrial carcinoma cells. Oncology Reports $\mathbf{2 8}$ 2035-2042. (doi:10.3892/or.2012.2038)

Yang L, Ravindranathan P, Ramanan M, Kapur P, Hammes SR, Hsieh JT \& Raj GV 2012 Central role for PELP1 in nonandrogenic activation of the androgen receptor in prostate cancer. Molecular Endocrinology 26 550-561. (doi:10.1210/me.2011-1101)

Received in final form 15 May 2014

Accepted 21 May 2014

Made available online as an Accepted Preprint

22 May 2014 http://erc.endocrinology-journals.org DOI: 10.1530/ERC-13-0502
(C) 2014 Society for Endocrinology Printed in Great Britain
Published by Bioscientifica Ltd 